\title{
A general vibration control methodology for human-induced vibrations
}

\author{
Xidong Wang $^{1} \mid$ Emiliano Pereira ${ }^{2}\left(\right.$ Jaime H. García-Palacios $^{1} \mid$ Iván M. Díaz $^{1}$
}

\author{
${ }^{1}$ ETSI Caminos, Canales y Puertos, \\ Universidad Politécnica de Madrid, \\ Madrid, Spain \\ ${ }^{2}$ Department of Signal Processing and \\ Communications, Universidad de Alcalá, \\ Madrid, Spain

\section{Correspondence} \\ Emiliano Pereira, Department of Signal \\ Processing and Communications, \\ Universidad de Alcalá, Madrid, Spain. \\ Email: emiliano.pereira@uah.es \\ Funding information \\ Research Net VIBRASTRUNET, \\ Grant/Award Number: \\ BIA2015-71942-REDT; China Scholarship \\ Council (CSC); Spanish project, \\ Grant/Award Number: DPI2013-47441
}

\begin{abstract}
Summary
This paper proposes a general methodology for the design of vibration control of human-induced vibrations. This uses a feedback loop for the design of control parameters and accounts for the nature of human excitation and how humans feel the vibration. The methodology developed considers not only single-input single-output control systems but also multi-input multi-output control systems, especially useful to cancel vibrations coming from modes with closely spaced natural frequencies. The strategy finds simultaneously the optimal placements of a set of inertial mass dampers and the design parameters (control gain matrix for active vibration control, damping ratios and tuning frequencies of tuned mass dampers for passive vibration control). To make this strategy implementable and suitable to human-induced vibrations, elements such as input-output frequency weighting, output time weighting, band-pass filters, actuator dynamics, and nonlinearities are considered within the design. The proposed methodology is illustrated in an application example on a realistic open-layout floor by designing single-input single-output and multi-input multi-output active and passive strategies with one and two dampers. Simulation tests are carried out to evaluate the performance of the controllers in terms of their vibration reduction capacity using a performance index indicator.
\end{abstract}

\section{KEYWORDS}

active control, feedback control, human-induced vibrations, passive control, vibration control

\section{1 | INTRODUCTION}

It is quite common nowadays to find vibration serviceability problems in lightweight and slender structures due to the developments of building materials and construction techniques. These structures satisfy ultimate limit state criteria but have the potential of attracting complaints coming from excessive human-induced vibrations. ${ }^{1,2}$ These problems can be found in footbridges, offices, concert halls, gyms, operating rooms, etc. Passive vibration control (PVC) and active vibration control (AVC) have been proved as efficient tools to control human-induced vibrations, ${ }^{3-5}$ in particular, when inertial mass dampers are used (tuned mass dampers, TMDs, and active mass dampers, AMDs).

Several AVC control strategies have been proposed up to now. An important group of strategies, see, for example, ${ }^{6,7}$ try to implement direct velocity feedback (DVF) using only one actuator. Single-input and single-output (SISO) AVC strategies have a good performance for controlling human-induced vibrations in a broad frequency bandwidth ${ }^{8}$; however, this might not work for a structure with closely spaced natural frequencies. Multi-input multi-output (MIMO) AVC strategies may solve this SISO AVC limitation. This issue was documented through simulation results by comparing ideal DVF SISO 
and MIMO controllers in Hanagan et al. ${ }^{9}$ However, the consideration of the actuator dynamics and the stability problems arisen from these dynamics must be considered in order to achieve successful practical implementations. ${ }^{10}$

Traditional TMD tuning only applies to single-TMD systems. ${ }^{11}$ The design of a multi-TMD system is not an easy task, because each TMD behaves like a perturbation to the others. Warnitchai and Hoang ${ }^{12}$ found a method, which can effectively handle a large number of design variables, to simultaneously search for the optimal placement and tuning of TMDs. Li et al. ${ }^{13}$ proposed an optimization procedure, based on the minimization of maximum root-mean-square (RMS) acceleration of a footbridge, to determine the optimal design parameters of a multi-TMD system.

The consideration of a TMD system as feedback controller simplifies the tuning of its parameters because active control design methodologies can then be applied. Examples of multi-TMD designs can be found in the previous studies. ${ }^{14-17}$ However, these contributions are not orientated to human-induced vibration, focusing on other vibration problems, like wind-induced vibration or earthquakes. Examples of designing single-TMD as a closed-loop system for human-induced vibration can also be found. In Casado et al., ${ }^{4}$ the methodology proposed in Poncela et al. ${ }^{18}$ is used to design a single-TMD for a footbridge application. However, a design methodology for designing multi-TMD for human-induced vibrations based on feedback control has not been proposed yet in the literature.

The proposed work generalizes the design methodology proposed in Pereira et al. ${ }^{10}$ by considering and extending the preliminary results published in the previous studies ${ }^{19,20}$ The main contribution is to propose a methodology to damp vibrations induced by human that can be used for active, semi-active, and/or passive inertial-mass-dampers systems. In addition, unlike the study of Pereira et al., ${ }^{10}$ this work shows how to consider in the design process of the damping system, the force input weighting, which reduces the dependence of the results respect to the disturbance and the nonlinearities presented in the inertial dampers (both AMD and TMD). Finally, this work also shows how to include output time weighting, output frequency weighting, band-pass filters, and AMD dynamics. ${ }^{10}$ All these aforementioned elements play an important role in practical implementations and should be considered within the design methodology.

This paper is organized as follows. Section 2 explains this common framework and the general control scheme. In addition, this section also includes the main elements for TMD and AMD systems and the general design process. Section 3 shows an application example of an open-plan floor model based on an existing dining room (with plenty of closely spaced modal frequencies) to demonstrate the usefulness of the presented methodology. Finally, some concluding remarks and future works are presented in Section 4.

\section{2 | FEEDBACK-BASED CONTROL FRAMEWORK}

The objective of this feedback-based control framework is to separate the structure dynamics from the damping system, which is treated like a feedback control. In other words, the structure applies forces to any system placed on it, and the system placed on the structure applies forces to the structure. The advantages of this feedback-based control methodology are to (a) unify designing criteria in order to tune different damping systems (like TMD or AMD), (b) simplify the combination of different damping systems into a single design method, (c) consider any linear/nonlinear model for damping systems without modifying the identified model of the structure, (d) reduce the computational cost needed to achieve a suitable solution, and (e) take advantage of the control theory (design methodologies, stability criteria, etc.) in order to design the damping system.

The general control scheme is shown in Figure 1 in which two main parts can be observed. The first one is the flexible structure dynamics, and the second one is the inertial mass control system. The flexible structure is modelled by $n$

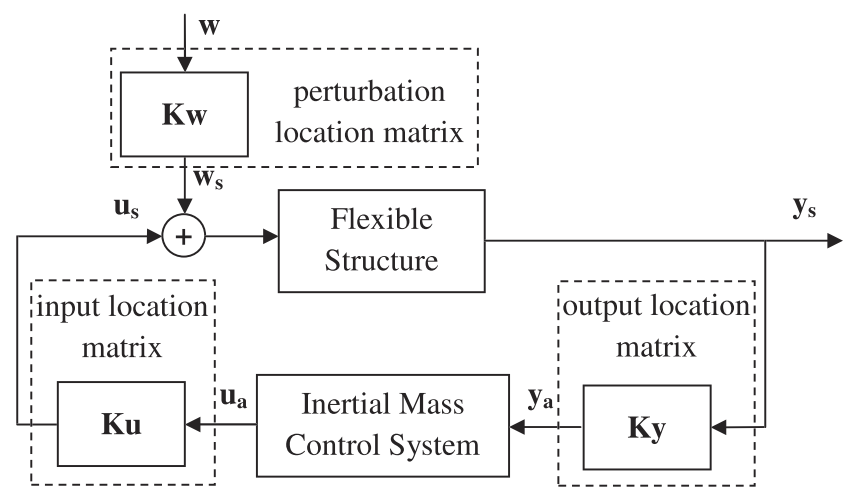


vibration modes and $p$ nodes (i.e., possible locations for TMDs/AMDs, sensors, and perturbations). The standard state-space representation can be as follows:

$$
\begin{aligned}
& \dot{\boldsymbol{x}}_{s}=\mathbf{A}_{s} \mathbf{x}_{s}+\mathbf{B}_{s_{u}} \mathbf{u}_{s}+\mathbf{B}_{S_{w}} \mathbf{w}_{s}, \\
& \mathbf{y}_{s}=\mathbf{C}_{s} \mathbf{x}_{s}+\mathbf{D}_{s_{u}} \mathbf{u}_{s}+\mathbf{D}_{s_{w}} \mathbf{w}_{s},
\end{aligned}
$$

in which $\mathbf{u}_{s}, \mathbf{w}_{s}$, and $\mathbf{y}_{s}$ are, respectively, the vectors formed by the controlled inputs (i.e., the force imparted by the control system), the perturbation forces, and acceleration measured at the locations, where accelerometers can be placed and/or human can feel the vibration. In addition, the matrices $\mathbf{A}_{s}, \mathbf{B}_{s_{u}}, \mathbf{B}_{s_{w}}, \mathbf{C}_{s}, \mathbf{D}_{s_{u}}$, and $\mathbf{D}_{s_{w}}$ define the complete state-space model of the structure. If Equation 1 is defined in modal coordinates, these state-space matrices are as follows ${ }^{21}$ :

$$
\begin{aligned}
& \mathbf{A}_{s}=\left[\begin{array}{ll}
\mathbf{0} & \mathbf{I} \\
-\Omega^{2} & -2 \mathbf{Z} \Omega
\end{array}\right], \quad \mathbf{B}_{s_{u}}=\mathbf{B}_{s_{w}}=\left[\begin{array}{l}
\mathbf{0} \\
\Phi
\end{array}\right], \\
& \mathbf{C}_{s}=\left[-\Omega^{2} \Phi^{T}-2 \mathbf{Z} \Omega \Phi^{T}\right], \mathbf{D}_{s_{u}}=\mathbf{D}_{s_{w}}=\Phi,
\end{aligned}
$$

in which $\boldsymbol{\Omega}$ and $\mathbf{Z}$ are, respectively, diagonal matrices formed by the angular natural frequencies $\left(\left[\omega_{1}, \ldots, \omega_{n}\right]\right)$ and damping ratios $\left(\left[\xi_{1}, \ldots, \xi_{n}\right]\right)$, respectively, and $\boldsymbol{\Omega}$ is the modal matrix with dimension $n \times p$. Note that each $k^{\text {th }}$ column of the latter matrix is formed by the $k^{\text {th }}$ vibration mode shape. The state vector is defined as follows: $\mathbf{x}_{s}=\left[x_{S_{1}}, \ldots, x_{s_{n}}, x_{s_{n+1}}, \ldots, x_{s_{2 n}}\right]$, $\left[x_{s_{1}}, \ldots, x_{s_{n}}\right]$ being the modal coordinates of the structure and $\left[x_{s_{n+1}}, \ldots, x_{s_{2 n}}\right]=\left[\dot{x}_{s_{1}}, \ldots, \dot{x}_{s_{n}}\right]$, their derivatives.

This general control scheme includes three location matrices, which simplify the perturbation model and the way of finding the optimal location for TMDs/AMDs and sensors. These matrices are defined as follows (see Figure 1):

$$
\mathrm{Ku}=\left[\begin{array}{lll}
K u_{11} & \ldots & K u_{1 q} \\
\vdots & \vdots & \vdots \\
K u_{p 1} & \ldots & K u_{p q}
\end{array}\right], \mathrm{Ky}=\left[\begin{array}{lll}
K y_{11} & \ldots & K y_{1 p} \\
\vdots & \vdots & \vdots \\
K y_{r 1} & \ldots & K y_{r p}
\end{array}\right], \mathrm{Kw}=\left[\begin{array}{lll}
K w_{11} & \ldots & 0 \\
\vdots & \ddots & \vdots \\
0 & \ldots & K w_{p p}
\end{array}\right]
$$

in which $q$ and $r$ are the number of actuators and sensors, respectively. Note that (a) the $j^{\text {th }}$ actuator is placed at the $l^{\text {th }}$ node when $K u_{l j}=1$, (b) the $i^{t h}$ sensor is placed at the $l^{\text {th }}$ node when $K y_{i l}=1$, and (c) there is a perturbation at the $l^{\text {th }}$ node when $K w_{l l}=1$. The control system can be a TMD (see Figure 2) or an AMD (see Figure 3) system. The general methodology proposed in this work consists of optimizing the Inertial Mass Control System (see Figure 1) and the location matrices by

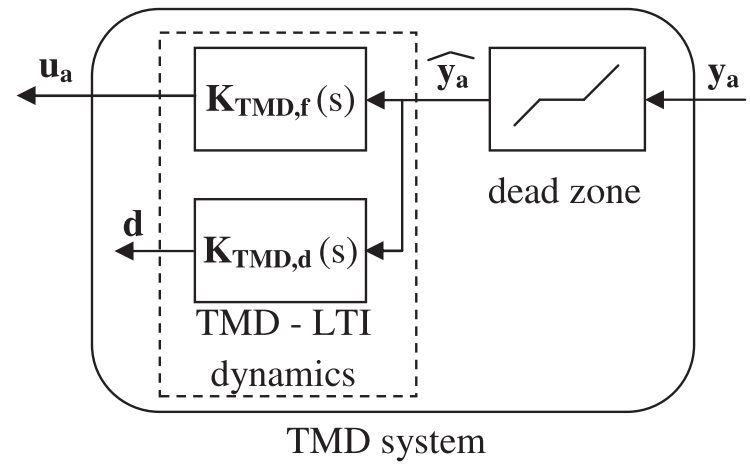

FIGURE 2 General tuned mass damper system (passive or semiactive) and linear and time invariant dynamics approximation

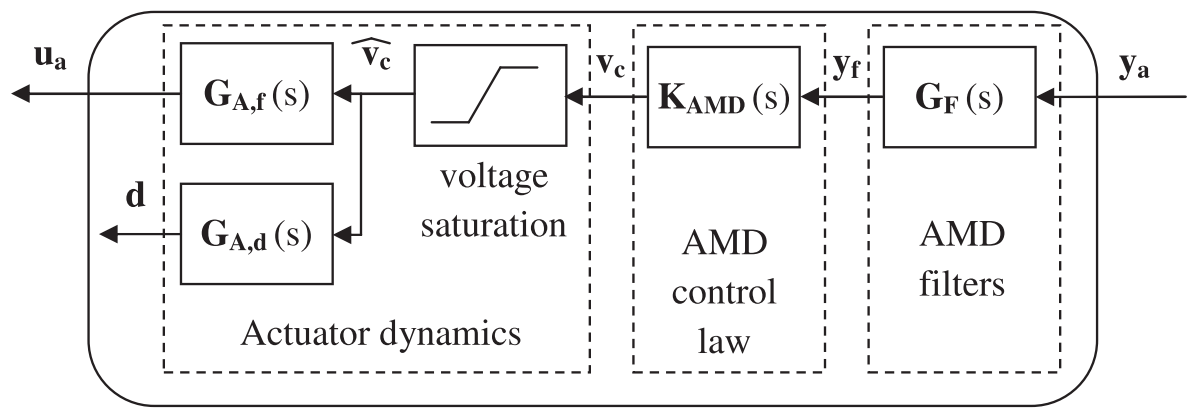

AMD system

FIGURE 3 Active mass damper system 
minimizing/maximizing a performance index indicator that considers the nature of the human loading and the perception to vibration. Note that TMD can be a passive or a semiactive system, whose model may consider nonlinearities and/or time variant parameters.

It should be remarked at this point that the methodology allows to excite the structure through both, by considering initial conditions of the flexible structure (i.e., $\mathbf{x}_{s}$ in $t=0$, where $t$ is the time variable) and/or a perturbation force $\left(\mathbf{w}_{s}\right.$ and text $K w$ ). This excitation should account for the structure common loads and the serviceability (considering the recomendable vibration levels for footbridges, offices, concert halls, gyms, or operating rooms are different). In addition, it should also be noted that, although the methodology does not include explicitly the actuator dynamics and AMD filters into the optimization (remind they are included into the feedback design methodology), they might be considered easily as a part of the AMD control law, allowing the optimization of the actuator properties and/or the filters.

\section{1 | TMD system}

A TMD can be modelled as a single-degree-of-freedom system (see Figure 4), where the force exerted on the structure $\left(u_{a, j}\right)$ depends on the relative movement between the TMD mass $\left(m_{t, j}\right)$ and the structure, damping, and stiffness. The "linear" acceleration input of the $j^{\text {th }}$ TMD located at $l^{\text {th }}$ node $\left(\hat{y}_{a, l}\right)$ is modelled as follows:

$$
\begin{array}{r}
\hat{y}_{a, l}=0 \quad \text { if } \quad\left|y_{a, l}\right|<y_{a, \min } \\
\hat{y}_{a, l}=y_{a, l}-y_{a, \min } \quad \text { if } \quad y_{a, l} \geq y_{a, \min } \\
\hat{y}_{a, l}=y_{a, l}+y_{a, \min } \quad \text { if } \quad y_{a, l} \leq-y_{a, \min },
\end{array}
$$

in which $y_{a, \min }$ is the minimum structure acceleration in absolute value for the TMD to start working. If the TMD linear stiffness and damping coefficient of the $j^{\text {th }}$ TMD are defined as $k_{t, j}$ and $c_{t, j}$, respectively, the transfer function (TF) between the force exerted by the $j^{\text {th }}$ TMD, and the linear acceleration of the $l^{\text {th }}$ structure node is as follows ${ }^{16}$ :

$$
\frac{U_{a, j}(s)}{\hat{Y}_{a, l}(s)}=-\frac{m_{t, j}\left(c_{t, j} s+k_{t, j}\right)}{m_{t, j} s^{2}+c_{t, j} s+k_{t, j}}=-m_{t, j} \frac{2 \xi_{t, j} \omega_{t, j} s+\omega_{t, j}^{2}}{s^{2}+2 \xi_{t, j} \omega_{t, j} s+\omega_{t, j}^{2}}=H_{T, f_{j}}(s),
$$

in which $\omega_{t, j}=\sqrt{k_{t, j} / m_{t, j}}$ and $\xi_{t, j}=c_{t, j} / 2 \sqrt{k_{t, j} m_{t, j}}$ are the angular natural frequency and damping ratio of the $j^{\text {th }}$ TMD as an isolated system, respectively, and $U_{a, j}(s)$ and $\hat{Y}_{a, l}(s)$ are the Laplace transform of $u_{a, j}$ and $\hat{y}_{a, l}$, respectively, $s$ being the Laplace variable. The TF between TMD mass displacement $\left(d_{j}\right)$ and the acceleration of the $l^{\text {th }}$ structure node can be obtained from Equation 5 as follows:

$$
H_{T, d_{j}}(s)=\frac{D_{j}(s)}{\hat{Y}_{a, l}(s)}=\frac{1}{s^{2} m_{t, j}} H_{T, f_{j}}(s)
$$

in which $D_{j}(s)$ is the Laplace transform of $d_{j}$.

The TMD model defined in Figures 2 and 4 considers the significant (linear) dynamics and nonlinearities (dead zone and stroke saturation). Note that the simulation used to obtain the optimum design parameters gives information about the maximum mass displacement of each TMD $\left(d_{j}\right)$, which can be used to select the appropriate TMD or to introduce a constraint associated to the maximum stroke.

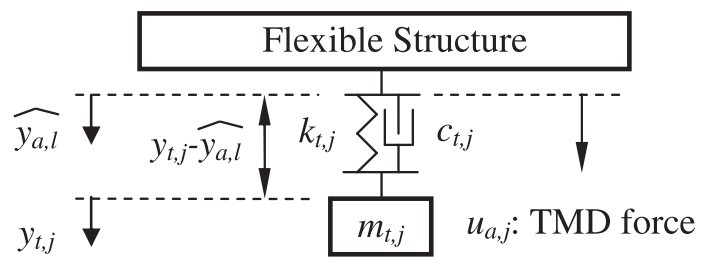


Finally, TMDs may be interpreted as systems, where the sensor and the actuator are placed at the same node (i.e., the location matrices $\mathrm{Ku}$ and $\mathrm{Ky}$ are diagonal). Therefore, the $q \times q($ or $r \times r$, since $q=r$ ) matrices of the TMD system (see Figure 2) are as follows:

$$
\mathbf{K}_{\mathbf{T M D}, \mathbf{f}}(s)=\left[\begin{array}{lll}
H_{T, f_{1}}(s) & \ldots & 0 \\
\vdots & \ddots & \vdots \\
0 & \ldots & H_{T, f_{q}}(s)
\end{array}\right], \quad \mathbf{K}_{\mathbf{T M D}, \mathbf{d}}(s)=\left[\begin{array}{lll}
H_{T, d_{1}}(s) & \ldots & 0 \\
\vdots & \ddots & \vdots \\
0 & \ldots & H_{T, d_{q}}(s)
\end{array}\right]
$$

The proposed methodology tunes the TMD parameters (typically $c_{t}$ and $k_{t}$ ) and the location matrices Ku and Ky (being $\mathrm{Ku}=\mathrm{Ky}^{T}$ for this particular case), also considering the dead zone and the maximum stroke, as it has been commented above.

\section{2 | AMD system}

The AMD system can be splitted into (a) AMD filters, (b) AMD control law, and (c) actuator dynamics (see Figure 3). Note that the control action is divided in two parts; one is going to be designed as control law, and the other includes filters to make the control law implementable. ${ }^{22}$ The following subsections will describe these components.

\subsection{1 | AMD filters}

The flexible structure model showed in Figure 1 and defined in Equations 1 and 2 considers the system output $\left(\mathbf{y}_{s}\right)$ as the acceleration measured at each node. This acceleration, which is usually obtained by accelerometers, must be conditioned before the "AMD control law" subsystem. This signal conditioning dynamics can be the corresponding of (a) the hardware used to measure the acceleration, which is usually neglected, (b) a low-pass filter, which is usually included to reduce the risk of spillover problems, ${ }^{23}$ and (c) a high-pass filter, which is usually used to reduce the risk of stroke saturation due to the effect of DC components of the measured signal and the low-frequency noise (see for example, the study of Wang et al. ${ }^{24}$ ). If the cutoff frequencies of these filters are chosen according to the resonant frequency of the actuator and the frequency bandwidth of the structure, then these cutoff frequencies may not be considered as design variables. Thus, on the one hand, the cutoff frequencies of the high-pass filters (denoted by $\omega_{H P}$ ) is the result of a tradeoff between the resonance frequency of actuator, because small values of $\omega_{H P}$ increase the risk of stroke saturation, and the first vibration mode of the structure, because higher values of $\omega_{H P}$ may reduce the damping imparted by the controller (see for example, the study of Pereira et al., ${ }^{10}$ where a DVF control is implemented). On the other hand, the cutoff frequencies of the low-pass filters (denoted by $\omega_{L P}$ ) must be sufficiently high when compared with the maximum frequency of the vibration modes that are expected to be controlled.

The $r \times r$ matrix of the AMD filters (see Figure 3) is as follows:

$$
\mathbf{G}_{\mathbf{F}}(s)=\left[\begin{array}{lll}
G_{F}(s) & \ldots & 0 \\
\vdots & \ddots & \vdots \\
0 & \ldots & G_{F}(s)
\end{array}\right]
$$

\subsection{2 | Actuator dynamics}

The actuator considered is an inertial mass actuator that generates forces through acceleration of an inertial mass to the structure on which it is placed. The actuator consists of an inertial (or moving) mass (denoted as $m_{A}$ ) attached to a current-carrying coil moving in a magnetic field created by an array of permanent magnets. The inertial mass is connected to the frame by a suspension system, modelled by a spring stiffness $k_{A}$ and a viscous damping $c_{A}$. Figure $5 \mathrm{a}$ shows an electrodynamic inertial actuator sketch and an example of a commercial one. The $r \times r$ matrices of the shaker linear part are as follows:

$$
\mathbf{G}_{\mathbf{A}, \mathbf{f}}(s)=\left[\begin{array}{lll}
G_{A_{f}}(s) & \ldots & 0 \\
\vdots & \ddots & \vdots \\
0 & \ldots & G_{A_{f}}(s)
\end{array}\right], \quad \mathbf{G}_{\mathbf{A}, \mathbf{d}}(s)=\left[\begin{array}{lll}
G_{A_{d}}(s) & \ldots & 0 \\
\vdots & \ddots & \vdots \\
0 & \ldots & G_{A_{d}}(s)
\end{array}\right],
$$

in which TFs $G_{A_{f}}(s)$ and $G_{A_{d}}(s)$ are defined as follows:

$$
G_{A_{f}}(s)=\frac{g_{A} s^{2}}{\left(s^{2}+2 \xi_{A} \omega_{A} s+\omega_{A}^{2}\right)(s+\epsilon)}, \quad G_{A_{d}}(s)=\frac{1}{s^{2} m_{A}} G_{A_{f}}(s),
$$


FIGURE 5 Inertial mass actuator: (a) Sketch of typical electrodynamic inertial actuator and (b) APS Dynamic Model 400 Shaker

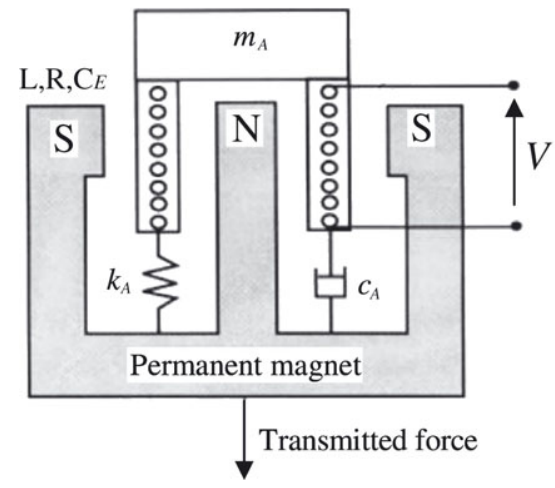

(a)

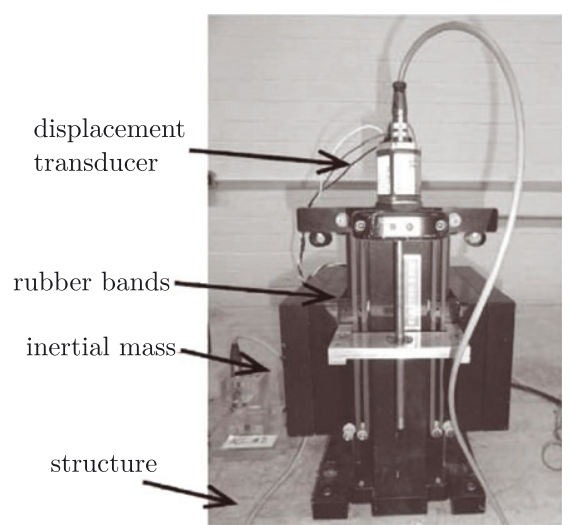

(b)

in which $g_{A}>0$ is the actuator gain parameter, $\xi_{A}$ is the damping ratio, $\omega_{A}$ is the angular natural frequency, and $\epsilon$ models the amplifier dynamics in voltage mode. If current mode for the amplifier is used, the term $(s+\epsilon)$ may be neglected, and the actuator damping ratio will be much smaller. ${ }^{25,26}$

\subsection{3 | AMD control law}

The AMD control law is defined by the following $q \times r$ matrix:

$$
\mathbf{K}_{\mathbf{A M D}}(s)=\left[\begin{array}{llll}
H_{A_{11}}(s) & H_{A_{12}}(s) & \ldots & H_{A_{1 r}}(s) \\
H_{A_{21}}(s) & H_{A_{22}}(s) & \ldots & H_{A_{2 r}}(s) \\
\vdots & \vdots & \ddots & \vdots \\
H_{A_{q 1}}(s) & H_{A_{q 2}}(s) & \ldots & H_{A_{q r}}(s)
\end{array}\right]
$$

The matrix $K_{A M D}(s)$ allows implementing any linear control law. For example, if each $H_{A_{i j}}(s)=-\gamma_{i j} / s$, this matrix represents the general case of a MIMO DVF.

\subsection{General design process}

The proposed design methodology is divided into the following steps:

a. Step 1: Dynamics identification. Identify the models of the flexible structure, inertial mass actuators, electronic equipments, etc.

b. Step 2: Filter design. Set the cutoff frequencies and filter types (Butterworth, Chebyshev, etc.) together with their orders, defining thus the matrix of Equation 8.

c. Step 3: Define the performance index (PI) to be mimized/maximized. This PI should consider the human vibration perception and excitation characteristics and be related to a magnitude representative of the structure serviceability (recommended vibration levels for footbridges, offices, concert halls, gyms, or operating rooms are different).

d. Step 4: Optimization problem setting. That is (a) the ranges of the variables used to compute the PI are defined, and (b) the number of actuators and sensors and their possible locations (i.e., the dimension of the location matrices $\mathrm{Ku}$ and $\mathrm{Ky}$ ) are defined. The selection of a reduced set of possible locations, which are candidates for the final placement, simplifies and makes computationally affordable the next step. This selection can be done from the mode shapes and frequencies of the structure.

e. Step 5: Resolution of the optimization problem. Find the optimal design for the matrices $K_{T M D}(s)$ or $K_{A M D}(s)$ by using a computational optimization algorithm (gradient-based method, genetic algorithms, etc.). The optimum location matrices ( $\mathrm{Ku}$ and $\mathrm{Ky})$ are also a result of this step.

This process will be carefully applied to an example in the following section. 

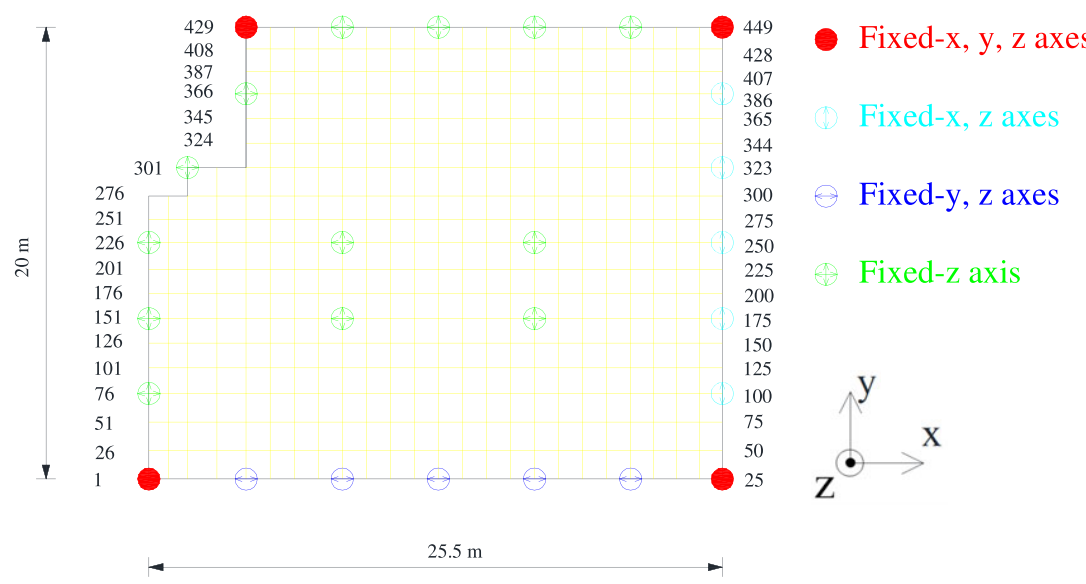

\section{3 | APPLICATION EXAMPLE}

FIGURE 6 Floor structure considered

The general design process is particularized for the design of two-TMD and two-AMD systems for a complex layout floor model based on a dining room floor of a primary-secondary school sited in Madrid (Spain). In addition, an evaluation of controller performances is included to show the advantages of using TMD or AMD with one (SISO) or two devices (MIMO).

\subsection{Step 1: Dynamics identification}

First of all, Figure 6 shows the layout of the floor structure and the general arrangement of beams and columns. A finite element (FE) model was created in ANSYS Multiphysics, ${ }^{28}$ using shell elements and 449 nodes. It is an almost rectangular floor with the dimension of $25.5 \times 20 \times 0.3 \mathrm{~m}$. As shown in Figure 6, the floor is considered as a continuous slab supported by 27 columns. Different connections between the floor and columns are marked with different colors: Red ones represent those whose displacements in $\mathrm{x}, \mathrm{y}$, and $\mathrm{z}$ directions are all restricted; cyan ones that are restricted in $\mathrm{x}$ and $\mathrm{z}$ displacements; blue ones that are restricted in $\mathrm{y}$ and $\mathrm{z}$ displacements; and green ones are connections only restricted in $\mathrm{z}$ direction. None of the connections between columns and floor are restricted in rotations. The yellow lines show the meshing grids of the shell elements. The material properties considered are modulus of elasticity $E=20 \times 10^{9} \mathrm{~N} / \mathrm{m}^{2}$, Poisson's ratio $v=0.15$, and density $\rho=3000 \mathrm{~kg} / \mathrm{m}^{3}$. The density has been increased from 2500 to $3000 \mathrm{~kg} / \mathrm{m}^{3}$ in order to include a portion of the imposed load (approximately 30\%) and the total dead load, following the recommendation of Smith et al. ${ }^{29}$ for analysis of floor vibrations. The mode shapes, angular natural frequencies, and modal masses of the first 10 vibration modes can be seen in Figure 7. The damping ratio of all these modes is assumed 0.01 .

The actuator considered in this work is an APS Dynamics model 400 electrodynamic shaker, which is shown in Figure 5b. The identified parameters of Equation $10 \mathrm{are}^{7} \omega_{A}=13.2 \mathrm{rad} / \mathrm{s}(2.1 \mathrm{~Hz}), g_{A}=12000$, and $\varepsilon=47.1$. Finally, the TMD model is assumed LTI as Equation 5, where the mass of each TMD is chosen as $1000 \mathrm{~kg}$.

\subsection{Step 2: Filters design}

A second-order Butterworth filter is considered for both high-pass and low-pass filters, and the cutoff frequencies adopted are $\omega_{H P}=2 \pi \cdot 2 \mathrm{rad} / \mathrm{s}$ for the high-pass filter and $\omega_{L P}=2 \pi \cdot 20 \mathrm{rad} / \mathrm{s}$ for the low-pass filter. So, the effect of both filters can be considered negligible for the frequency bandwidth of the first 10 vibration modes. The TF $G_{F}(s)$ of each element of matrix defined in Equation 8 is as follows:

$$
G_{F}(s)=\frac{\omega_{H P}^{2} S^{2}}{s^{2}+(2 / \sqrt{2}) \omega_{H P} S+\omega_{H P}^{2}} \cdot \frac{\omega_{L P}^{2}}{s^{2}+(2 / \sqrt{2}) \omega_{L P} S+\omega_{L P}^{2}} .
$$

\section{3 | Step 3: Define the PI}

The objective is to define a modified PI based on previous PI proposed in the previous studies. ${ }^{9,10}$ Like in those studies, ${ }^{9,10}$ the state vector $\mathbf{x}_{s}$ is considered in order to defined the PI. In addition, the initial conditions of the state space model defined into Equation 1 are used to define the system perturbation. Finally, it should be highlighted that the PI does not modify the control loop. Therefore, it does not affect to the stability of the control system. 
Modal mass: 46.13 tons

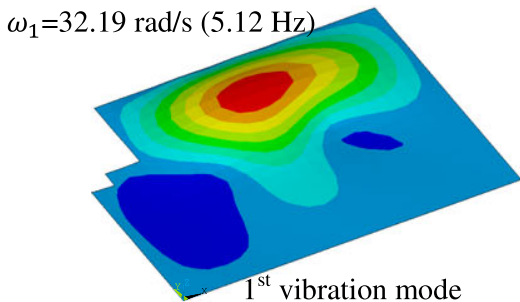

Modal mass: 52.87 tons

$\omega_{3}=41.79 \mathrm{rad} / \mathrm{s}(6.65 \mathrm{~Hz})$

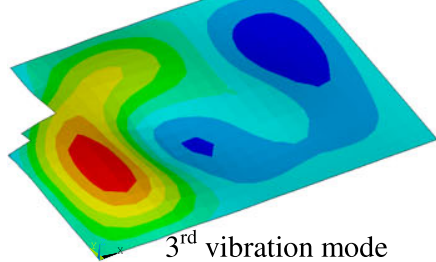

Modal mass: 54.41 tons

$\omega_{5}=48.13 \mathrm{rad} / \mathrm{s}(7.66 \mathrm{~Hz})$

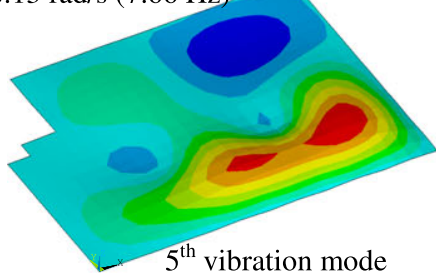

Modal mass: 73.05 tons

$\omega_{7}=62.97 \mathrm{rad} / \mathrm{s}(10.02 \mathrm{~Hz})$

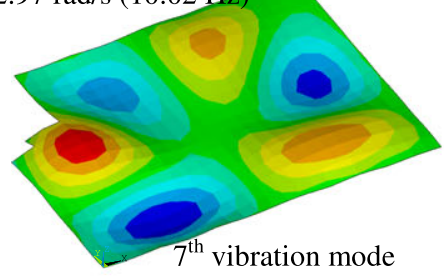

Modal mass: 64.91 tons

$\omega_{9}=78.09 \mathrm{rad} / \mathrm{s}(12.43 \mathrm{~Hz})$

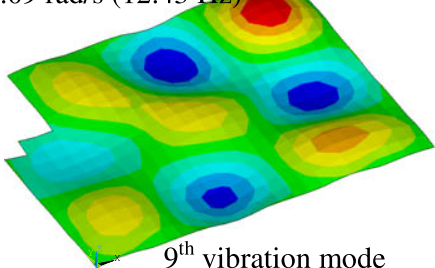

Modal mass: 67.05 tons

$\omega_{2}=36.18 \mathrm{rad} / \mathrm{s}(5.76 \mathrm{~Hz})$

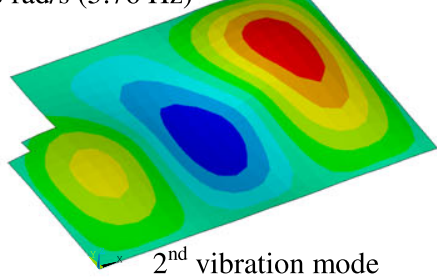

Modal mass: 85.90 tons

$\omega_{4}=47.51 \mathrm{rad} / \mathrm{s}(7.56 \mathrm{~Hz})$

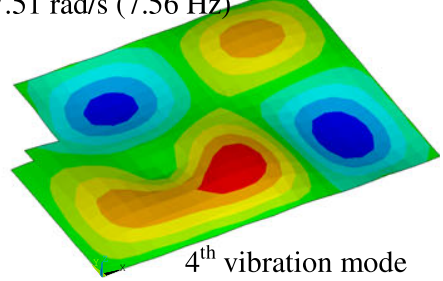

Modal mass: 49.85 tons

$\omega_{6}=58.61 \mathrm{rad} / \mathrm{s}(9.33 \mathrm{~Hz})$

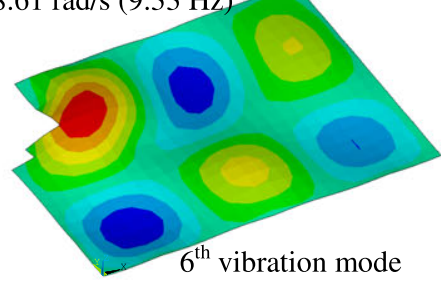

Modal mass: 36.56 tons

$\omega_{8}=69.32 \mathrm{rad} / \mathrm{s}(11.03 \mathrm{~Hz})$

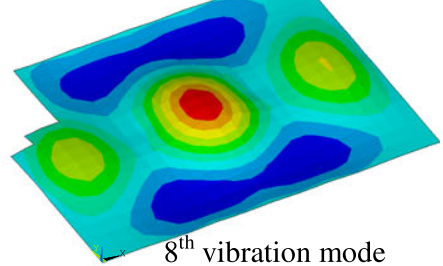

Modal mass: 54.62 tons

$\omega_{10}=85.19 \mathrm{rad} / \mathrm{s}(13.56 \mathrm{~Hz})$

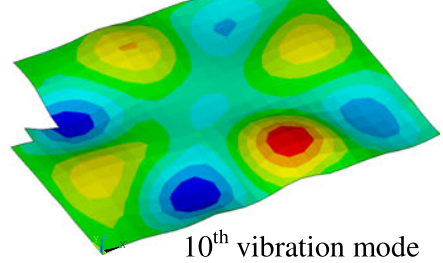

FIGURE 7 Floor mode shapes, angular natural frequencies, damping ratios, and modal masses 
The human perception depends on the amplitude, frequency, and duration of the vibration. The amplitude and frequency are considered herein by the frequency weighting functions defined in $\mathrm{ISO}^{27}$ and $\mathrm{BS}^{2} 841 .^{30}$ According to ISO, ${ }^{27}$ for z-axis vibration and standing and seating, the frequency weighting function is $W_{k}$ (denoted here as $g_{\text {OFW }}$ ), which corresponds with a filter with the frequency response shown in Figure 8.

The duration of the vibration, which affects human comfort, ${ }^{31}$ is also weighted in the proposed control design by multiplying the system response by an exponential time weighting of the form $e^{\alpha t}$. Note that this $\alpha>0$ is analogous to add a constraint in the relative stability of the controlled system because the transient response of any linear time invariant system is a function multiplied by a sum of exponential of the form $e^{-\omega_{i} \xi_{i} t}$, where $-\omega_{i} \xi_{i}$ is the real part of the $\mathrm{i}^{\text {th }}$ vibration mode. Thus, the persistent states are penalized more heavily as $\alpha$ is increased. The value of $\alpha$ is usually considered as the maximum value of the real part corresponding to open-loop system poles. Thus, the controller must increase the relative stability of the closed-loop system in order to be considered.

The human vibration perception is considered in the controller design by weighting the state vector of the structure $\mathbf{x}_{s}$ by the following frequency and time weighting:

$$
x_{s w_{l}}=\left(e^{\alpha t} x_{s_{l}}(t)\right) * g_{\text {OFW }}(t), l \in[1, \ldots, 2 n],
$$

in which $(*)$ denotes the convolution process, and $g_{O F W}(t)$ is the impulse response function of a system with the frequency response of Figure 8.

The human excitation is considered like in Hanagan et al., ${ }^{9}$ where a nonzero initial condition to the velocity states of the structure is introduced $\left(\mathbf{x}_{s}(0)=\left[0, \ldots, 0, x_{s_{n+1}}(0), \ldots, x_{s_{2 n}}(0)\right]^{T}\right)$ in order to approximate the influence of zero initial conditions and a spatially distributed, but temporally impulsive, disturbance force. However, unlike the previous studies, ${ }^{9,10}$ where all the vibration modes of the structure may be excited in the same way, this work proposed a novel modification based on considering the specific dynamic loading factor (DLF) of each vibration mode corresponding to a certain frequency range. ${ }^{32}$ Based on the DLFs analysis in vertical direction by Brownjohn et al., ${ }^{33}$ the following input weighting function is applied to weight the initial conditions:

$$
g_{I F W}(f)=\left\{\begin{array}{ll}
0.02 f & 0 \quad \mathrm{~Hz}<f<1.2 \mathrm{~Hz} \\
0.37 f-0.42 & 1.2 \mathrm{~Hz} \leq f<2.4 \mathrm{~Hz} \\
-0.003 f+0.06 & 2.4 \mathrm{~Hz} \leq f<20 \mathrm{~Hz} \\
0 & f \geq 20 \mathrm{~Hz}
\end{array},\right.
$$

in which $f$ is the frequency to be weighted. Thus, the initial condition of the modal coordinates is weighted according to corresponding modal frequencies as follows:

$$
x_{n+k}(0)=F_{0} \phi_{k, \max } g_{I F W}\left(f_{k}\right), k \in[1, \ldots, n],
$$

in which the value of $F_{0}$ is chosen as $2670 \mathrm{~N},{ }^{34}$ which is the normalized maximum force of an idealized heel drop excitation, and $f_{k}$ is the natural frequency of $k^{\text {th }}$ vibration mode. This input weighting function contributes interestingly to achieve optimum solutions, which are independent on the system perturbation adopted.

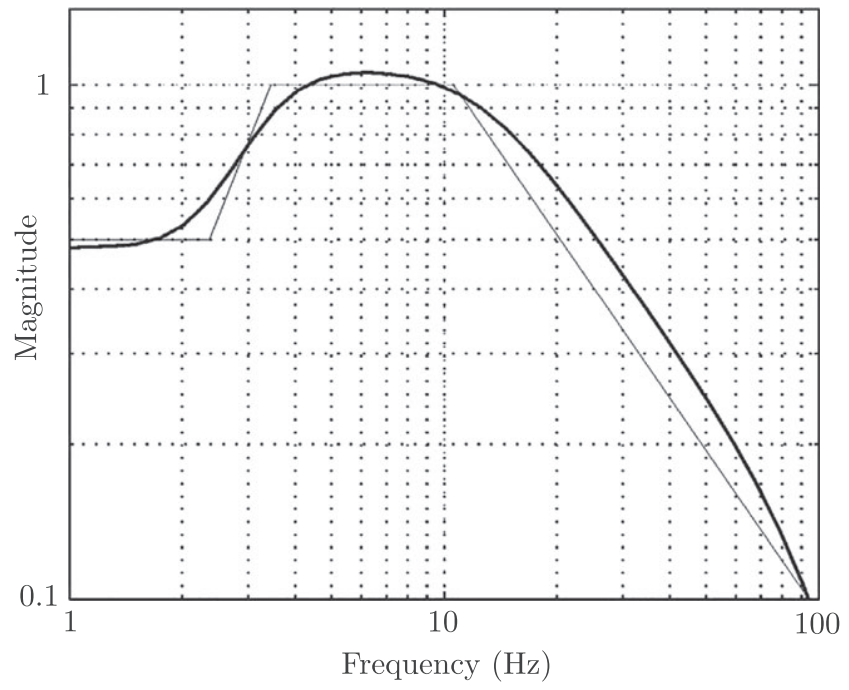

FIGURE 8 Frequency weighting function $g_{O F W}$ (thicker curve) and its asymptotic definition (thinner curve) ${ }^{27}$ 
The proposed PI considers the weighted state vector and the maximum value of each vibration mode shape (i.e., eigenvector). The PI is mathematically defined as follows:

$$
\operatorname{PI}(\mathbf{z})=\frac{1}{2} \int_{0}^{t_{f}} \mathbf{x}_{s w}^{T}(\mathbf{z}) \mathbf{Q} \mathbf{x}_{s w}(\mathbf{z}) \mathrm{d} t,
$$

in which the variable $\mathbf{z}$ includes the parameters of matrices $K_{T M D}(s)$ (for a TMD system) or $K_{A M D}(s)$ (for an AMD system), $\mathrm{Ku}$ and Ky. The matrix $\mathbf{Q}$ is a $2 n \times 2 n$ positive definite matrix, which is taken as follows ${ }^{9}$ :

$$
\mathbf{Q}=\left[\begin{array}{llllll}
\omega_{1}^{2} \phi_{1, \max }^{2} & \ldots & 0 & 0 & \ldots & 0 \\
\vdots & \ddots & \vdots & \vdots & \ddots & \vdots \\
0 & \ldots & \omega_{n}^{2} \phi_{n, \max }^{2} & 0 & \ldots & 0 \\
0 & \ldots & 0 & \phi_{1, \max }^{2} & \ldots & 0 \\
\vdots & \ddots & \vdots & \vdots & \ddots & \vdots \\
0 & \ldots & 0 & 0 & \ldots & \phi_{n, \max }^{2}
\end{array}\right]
$$

$\phi_{k, \text { max }}$ being the maximum value of the $k^{\text {th }}$ eigenvector $\phi_{k}$. Note that the displacement states are weighted by the natural frequencies, thus making the displacement states comparable with the velocity states. Note also that the PI is a measure of the total involved vibration energy accounting for the nature of the human perception and nature of human loading. Finally, the value of $t_{f}$ is the simulation time to obtain the performance index indicator, which must be large enough to achieve the steady state of $f(\mathbf{z})$ (i.e., the weighted state vector $\mathbf{x}_{s w} \cong \mathbf{0}$ ).

\subsection{Step 4: Define the input variables for the PI}

Before finding the value of $\mathbf{z}$, which minimizes the PI defined by Equation 16, the number of actuators/sensors and the possible locations are defined. In addition, the selection of possible candidate nodes might be firstly selected in order to reduce the computational cost. This first selection can be done, for example, by design experience of by a mathematical criterion. This example considers the $\mathrm{H}$-infinity norm and $\mathrm{H} 2$ norm calculation proposed in Gawronski. ${ }^{21}$ The nodes with the largest values of the following norms are chosen as the best candidate points from all the $p$ nodes of the structure:

$$
\begin{gathered}
H_{\infty}(l)=\sum_{m=1}^{p}\left\|\frac{Y_{S_{m}}(s)}{W_{S_{l}}(s)}\right\|_{\infty}, l, m \in[1, \ldots, p], \\
H_{2}(l)=\sum_{m=1}^{p}\left\|\frac{Y_{S_{m}}(s)}{W_{S_{l}}(s)}\right\|_{2}, l, m \in[1, \ldots, p],
\end{gathered}
$$

in which $H_{\infty}(l)$ and $H_{2}(l)$ represent the sum of the $p$ corresponding norms (H-infinity and $\mathrm{H} 2$, respectively) of TFs, between acceleration response at all considered nodes $\left(Y_{S_{m}}(s)\right)$ and the force at the $l^{\text {th }}$ node $\left(W_{S_{l}}(s)\right)$. Note that given a certain force at these candidate nodes, both $\mathrm{H} 2$ norm and $\mathrm{H}$-infinity norm of all the $n$ vibration modes are the highest. Hence,

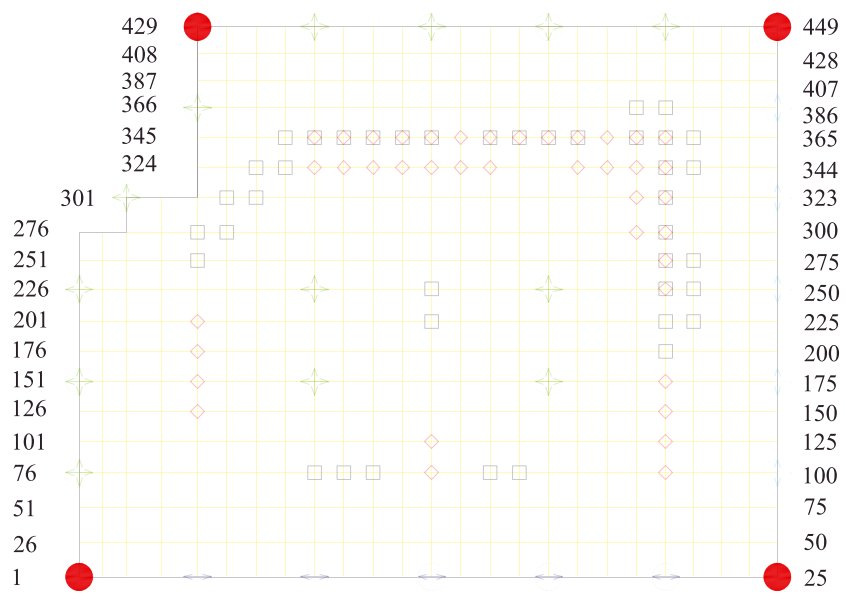

$$
\text { H_infinity indicators } \square \text { H_2 indicators }
$$


these selected points are the best candidates to place the actuators and/or TMDs. Thus, the 449 nodes considered initially are reduced to 16 points (see Figure 9), which lessens the computational cost by $96.44 \%$ and $99.88 \%$ for control systems consisting of $1 \mathrm{TMD} / \mathrm{AMD}$ and 2 TMDs/AMD, respectively. Note that, in this application example, collocated control is assumed for AMD system. Then, the number of sensor and actuators are the same $(q=r)$, and they are located at the same place.

\subsection{Step 5: Find the optimal design for the matrices $K_{T M D}(s)$ or $K_{A M D}(s), \mathrm{Ku}$, and $\mathrm{Ky}$}

This work uses the MATLAB function fminsearch, which uses the search method of Lagarias et al. ${ }^{35}$ This function is used to find the optimal values of $K_{T M D}(s)$ or $K_{A M D}(s)$ for the 16 candidate points obtained in previous design step. Thus, the following optimal design are firstly obtained:

- 16 optimal solutions for SISO TMD and AMD systems.

- 256 optimal solutions for MIMO TMD and AMD systems (two TMDs/AMDs).

Each optimal solution calculates the minimum value of Equation 16. The parameter $\alpha$ (time weighting) is defined by $\alpha=0.01 \cdot 35.19=0.3519$, which is the natural frequency of the first vibration mode multiplied by the considered structural damping for all nodes (0.01). Thus, the controlled system must be damped by the control system in order to be considered. In addition, the damper stroke conditions are also considered. The considered value for both TMDs/AMDs is $80 \mathrm{~mm}$, which is the maximum allowable stroke of the actuator, APS Dynamics Model 400 Shaker. Thus, if the selected parameters (by fminsearch) of $K_{T M D}(s)$ or $K_{A M D}(s)$ do not achieve these conditions, the value of $\mathrm{PI}(\mathbf{z})$ is penalized, rejecting this configuration. Finally, the best among all these optimal solutions is chosen.

Using the common framework, four control systems are designed: PVC of 1 TMD, PVC of 2 TMDs, AVC of 1 AMD, and AVC of 2 AMDs. As mentioned above, the mass of each TMD is equal to $1000 \mathrm{~kg}$. Therefore, the parameters to be

TABLE 1 Tuning parameters

\begin{tabular}{|c|c|c|}
\hline Damping system & $\begin{array}{l}\text { Optimised parameters of } \\
K_{T M D}(s) \text { and/or } K_{A M D}(s)\end{array}$ & $\begin{array}{l}\text { Number of terms of } \mathrm{Ku} \text { and/or } \mathrm{Ky} \\
\text { equal to } 1\end{array}$ \\
\hline $1 \mathrm{AMD}$ & $-\gamma_{11} / s$ & 2 \\
\hline 2 AMDs & {$\left[\begin{array}{ll}-\gamma_{11} / s & -\gamma_{12} / s \\
-\gamma_{21} / s & -\gamma_{22} / s\end{array}\right]$} & 4 \\
\hline 1 TMD & $-m_{t, 1} \frac{2 \xi_{t, 1} \omega_{t, 1} s+\omega_{t, 1}^{2}}{s^{2}+2 \xi_{t, 1} \omega_{t, 1} s+\omega_{t, 1}^{2}}$ & 1 \\
\hline 2 TMDs & {$\left[\begin{array}{cc}-m_{t, 1} \frac{2 \xi_{t, 1} \omega_{t, 1} s+\omega_{t, 1}^{2}}{s^{2}+2 \xi_{t, 1} \omega_{t, 1} s+\omega_{t, 1}^{2}} & 0 \\
0 & -m_{t, 2} \frac{2 \xi_{t, 2} \omega_{t, 2} s+\omega_{t, 2}^{2}}{s^{2}+2 \xi_{t, 2} \omega_{t, 2} s+\omega_{t, 2}^{2}}\end{array}\right.$} & 2 \\
\hline
\end{tabular}

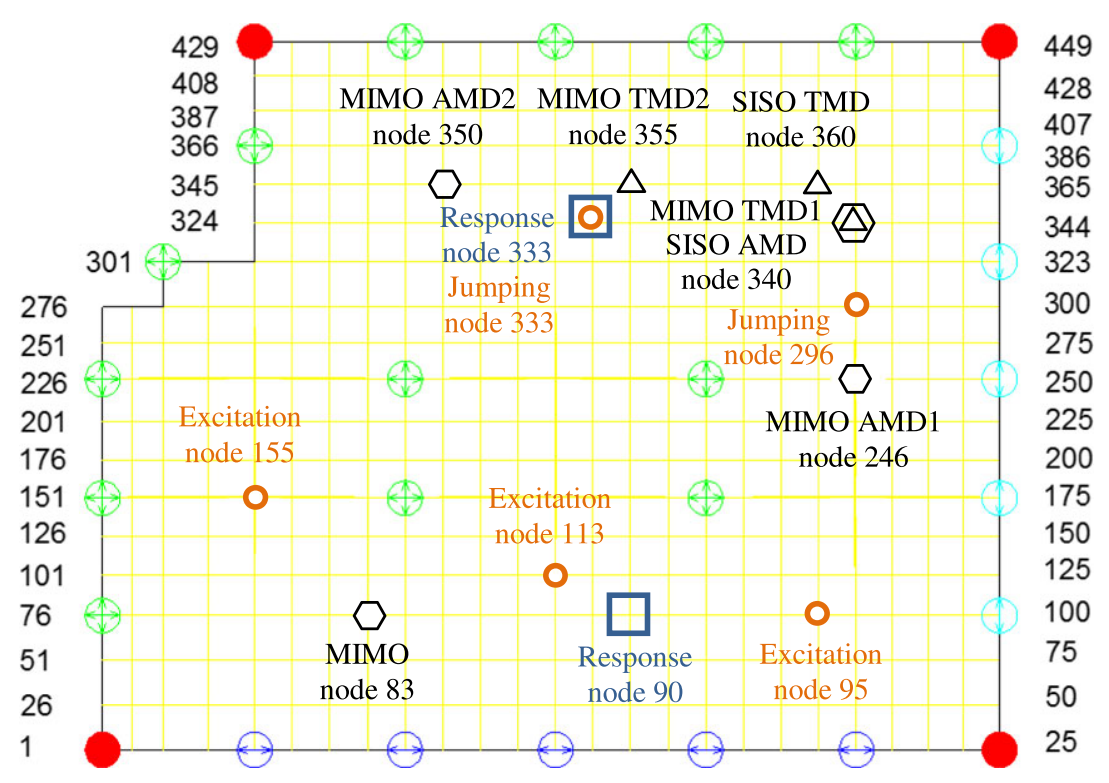

FIGURE 10 Optimum locations of tuned mass dampers (triangle) and active mass dampers (hexagons); excitation (circles) and response (squares) nodes 
tuned are the damping ratio $\left(\xi_{t, j}\right)$ and angular natural frequency $\left(\omega_{t, j}\right)$ of each TMD. The AMD considered in this work is a DVF, which involves the tuning of each gain $\left(\gamma_{i j}\right)$. In addition, the location matrices (Ku and Ky) are obtained. Table 1 summarizes the control system parameters. The optimal controller designs are the following:

- 1 AMD:

- Control parameters: $\gamma_{11}=2228.2, K_{u_{340,1}}=1$ and $K_{y_{1,340}}=1$.

- PI: $15.13 \times 10^{-6}$.

- 2 AMDs

- Control parameters: $\gamma_{11}=3738.3, \gamma_{12}=17.8, \gamma_{21}=-1236.0, \gamma_{22}=3638.1, K_{u_{246,1}}=1, K_{u_{350,2}}=1, K_{y_{1,246}}=1$ and $K_{y_{2,350}}=1$.

- PI: $9.80 \times 10^{-6}$.

- 1 TMD

- Control parameters: $\xi_{t, 1}=0.115, \omega_{t, 1}=34.571 \mathrm{rad} / \mathrm{s}, K_{u_{360,1}}=1$ and $K_{y_{1,360}}=1$.

- PI: $19.87 \times 10^{-6}$.

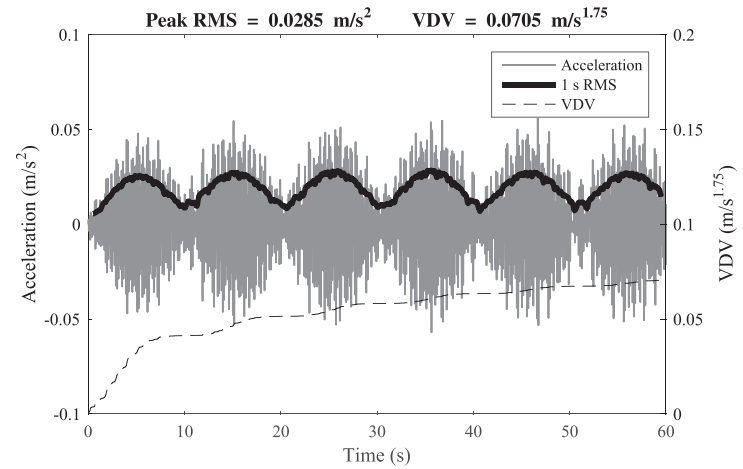

(a)

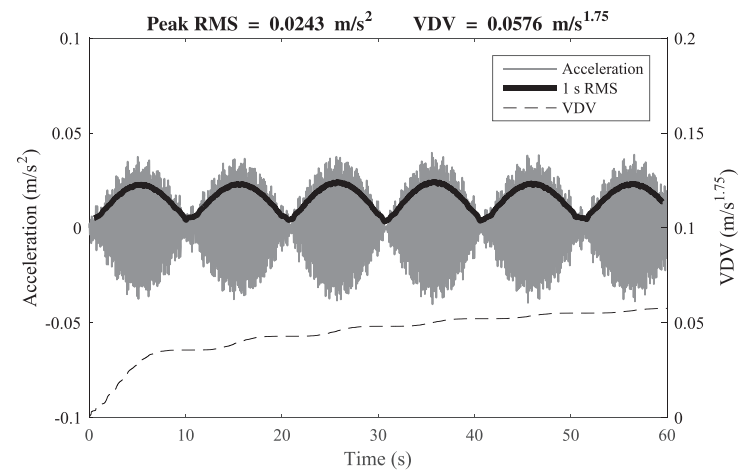

(c)

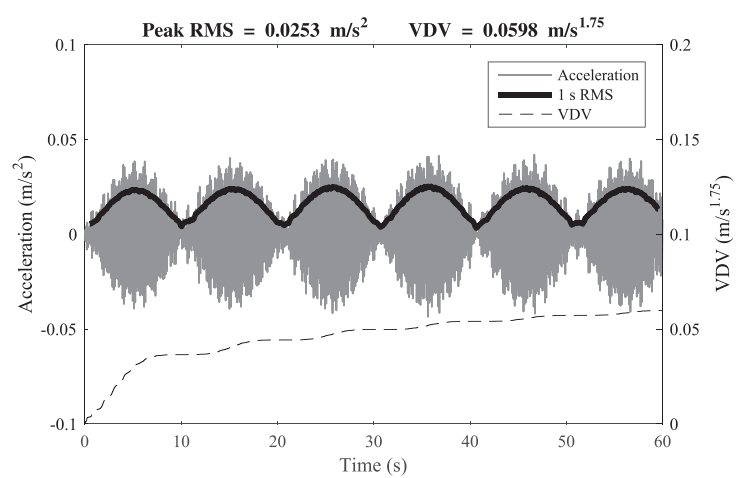

(b)

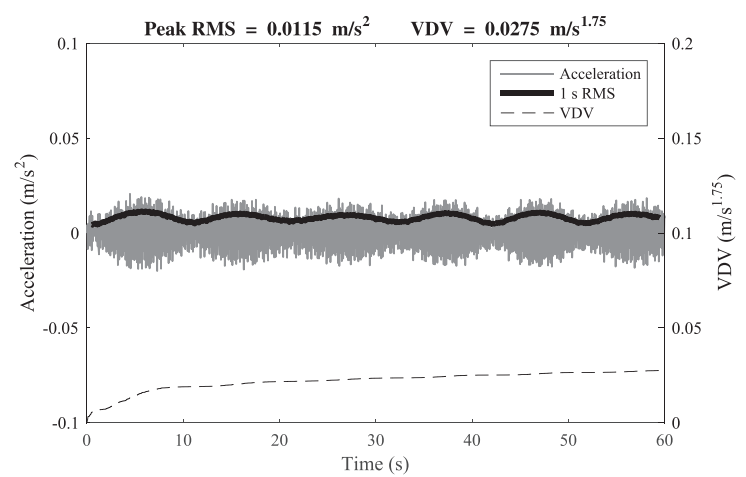

(d)

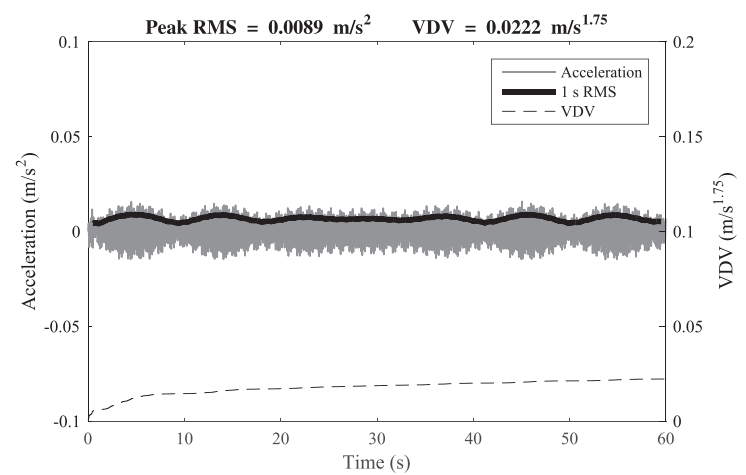

(e)

FIGURE 11 Time history response at Node 90 under human jumping excitation: (a) without control; (b) 1 TMD; (c) 2 TMDs; (d) 1 AMD; (e) 2 AMDs 
- 2 TMDs

- Control parameters: $\xi_{t, 1}=0.075, \omega_{t, 1}=39.323 \mathrm{rad} / \mathrm{s}, \xi_{t, 2}=0.096, \omega_{t, 2}=31.545 \mathrm{rad} / \mathrm{s}, K_{u_{340,1}}=1, K_{u_{35,2}}=1$, $K_{y_{1,340}}=1$ and $K_{y_{2,355}}=1$.

- PI: $14.02 \times 10^{-6}$.

Note that the optimal locations for dampers are not obvious and together with which the system parameters cannot be solved by traditional control design tuning methods. It is worthwhile to mention that comparing the performance between AVC and PVC is not purpose of this work.

\subsection{Evaluation of controller performances}

The performance of all the controllers is tested by exciting the structure with a jumping excitation. This perturbation is a representative example of periodic (jumping is a rhythmic human activity, as well as dancing or aerobics) loads, which can be generated by humans.

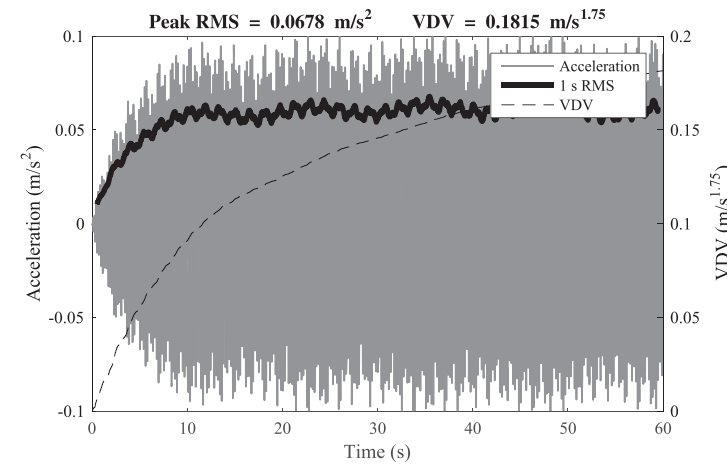

(a)

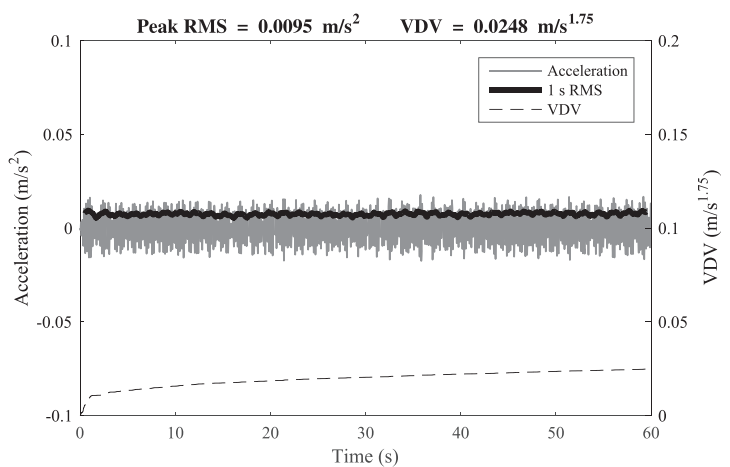

(c)

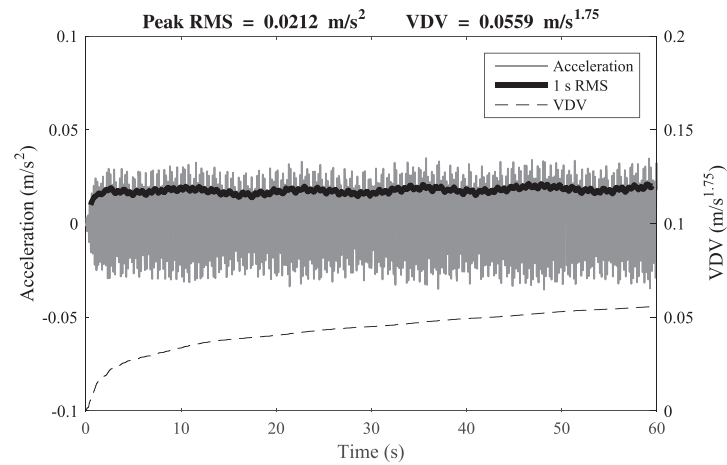

(b)

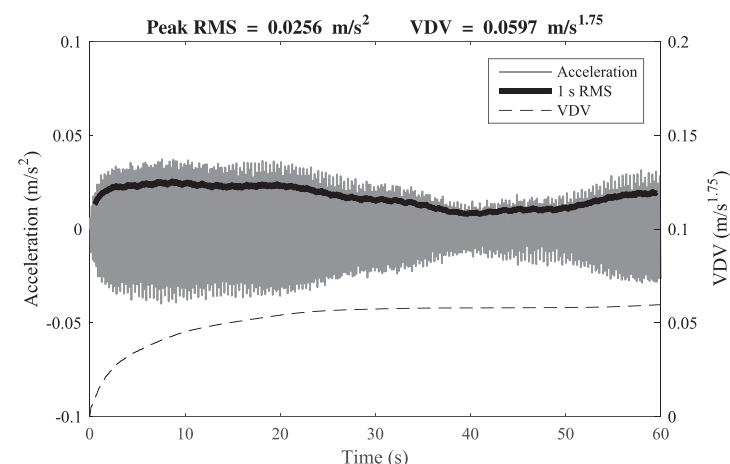

(d)

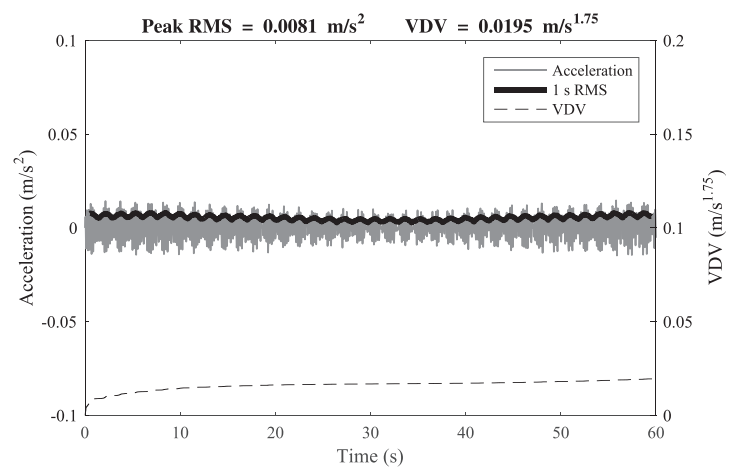

(e)

FIGURE 12 Time history response at Node 333 under human jumping excitation: (a) without control; (b) 1 TMD; (c) 2 TMDs; (d) 1 AMD; (e) 2 AMDs 
FIGURE 13 Percentage of floor area where a VDV value is exceeded

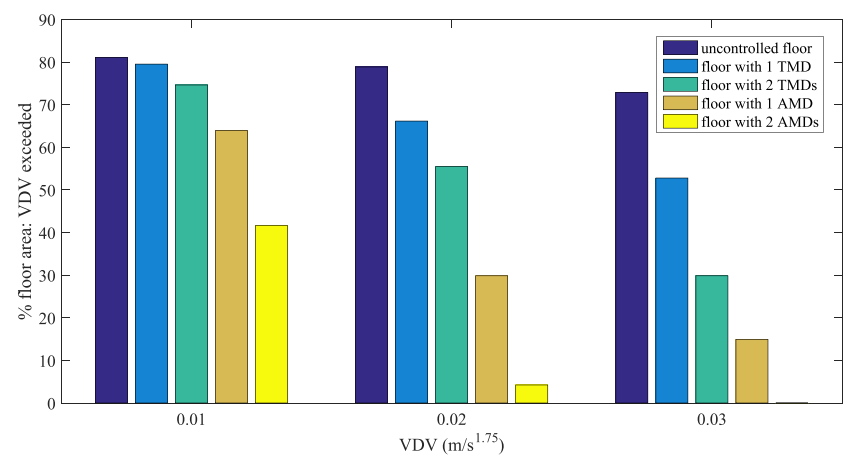

For the jumping excitation, a pedestrian of 800-N weight is considered, and the DLFs (amplitude of each harmonic) of the excitation proposed in $\mathrm{ISO}^{36}$ are assumed (the periodic excitation is represented as a Fourier series). Five nodes (333, 296, 155, 113, and 95) are chosen to excite (see Figure 10) with these frequencies: (a) $2.56 \mathrm{~Hz}$-Node 333-first vibration mode (5.12 Hz), (b) $1.92 \mathrm{~Hz}$-Node 296-second vibration mode $(5.76 \mathrm{~Hz})$, (c) $2.22 \mathrm{~Hz}$-Node 155—-third vibration mode (6.65 Hz), (d) $2.52 \mathrm{~Hz}$-Node 113-fourth vibration mode $(7.56 \mathrm{~Hz})$, and (e) $2.55 \mathrm{~Hz}$-Node 95-fifth vibration mode $(7.66 \mathrm{~Hz})$. It should be highlighted that the vibration mode shape value for the first five modes is maximum for the nodes $333,296,155,113$, and 95.

Figures 11 and 12 show the simulated time response at node 90 and node 333 for the five cases, respectively. The RMS computed each $1 \mathrm{~s}$ is also included in those figures and used it for performance evaluation. Nodes 90 and 333 show the vibration level in two different parts of the floor. Note that the vibration level at node 333 (RMS value is $0.0285 \mathrm{~m} / \mathrm{s}^{2}$ ) is larger than the one at node 90 (RMS value is $0.0678 \mathrm{~m} / \mathrm{s}^{2}$ ). This is due to the fact that the vibration level of the first two vibration modes is more important. Thus, vibration reduction should be significant at node 333 and pretty insignificant at node 90. With respect to AVC, AMDs can cancel more than one vibration mode; therefore, the optimum location should reduce the vibration level at both nodes. This is showed in Figures 11 and 12. The peak acceleration RMS at node 90 is the following: (a) $0.0253 \mathrm{~m} / \mathrm{s}^{2}$ (88.8 \%) for SISO TMD, (b) $0.0243 \mathrm{~m} / \mathrm{s}^{2}\left(85.3 \%\right.$ ) for MIMO TMD, (c) $0.0115 \mathrm{~m} / \mathrm{s}^{2}(40.4 \%)$ for SISO AMD, and (d) $0.0089 \mathrm{~m} / \mathrm{s}^{2}$ (31.2\%) for MIMO AMD. The peak acceleration RMS at node 333 is (a) $0.0212 \mathrm{~m} / \mathrm{s}^{2}(31.3$ \%) for SISO TMD, (b) $0.0095 \mathrm{~m} / \mathrm{s}^{2}$ (14.0\%) for MIMO TMD, (c) $0.0256 \mathrm{~m} / \mathrm{s}^{2}$ (37.8 \%) for SISO AMD, and (d) $0.0081 \mathrm{~m} / \mathrm{s}^{2}$ (12.0\%) for MIMO AMD.

The performance is also evaluated using the vibration dose value (VDV), also known as fourth power vibration dose method, which is used to provide information about the serviceability of the floor for rhythmic activities. ${ }^{29}$ Figures 11 and 12 show the running VDV for their particular location, and the final value is the one used for the analysis. The VDV of all nodes on the floor during $60 \mathrm{~s}$ of jumping excitation is calculated. Figure 13 shows the percentage of floor area, where a VDV value ( $\mathrm{x}$ axis) is exceeded. Note that Figure 13 better illustrates control objective of the above controllers, which is to reduce the vibration all over the floor area. In addition, it should be noted that there is not any node in which the VDV value of 0.03 is overtaken for MIMO AMD.

\section{4 | CONCLUSIONS}

This paper proposes a methodology for the design of inertial mass vibration control systems for human-induced vibrations. Thus, any vibration control system design (active, semiactive, and/or passive) can be carried out from the presented methodology. Moreover, this work proposes a step-by-step practical guideline in which the vibration control system considers how humans excite a structure and feel the vibrations simultaneously. In addition, the significant dynamics and nonlinearities (both AMD and TMD), such as high-pass filters, low-pass filters, voltage, and stroke saturation, which are extremely important for a successful practical implementation, are also considered into the design methodology and the guidelines. The work concludes with an application example of a realistic floor model, for which optimal SISO and MIMO TMDs/AMDs are designed and tested, thus showing the aforementioned advantages of the proposed common framework. Interesting results are reported: (a) when designing a MIMO AVC, a fully centralized algorithm is the optimal one, and the optimal location is not obvious in any way, (b) when TMDs are designed, they are strategically tuned design at frequencies between two vibration modes and placed at overlapping areas of mode shapes.

Because this methodology simplifies and facilitates the comparison between different vibration control systems, future works can consider the selection of different actuators, control laws, optimization algorithms, etc. in order to achieve a 
vibration serviceability goal of the structures including additionally economic factors. Moreover, the inclusion of actuator parameters regarding AVC, together with the control law optimization, can be considered in the future.

\section{ACKNOWLEDGEMENTS}

The work in this paper is part of the Spanish project DPI2013-47441: Development of new systems for reducing vibrations in pedestrian structures. The authors also acknowledge the partial financial support provided by the Research Net VIBRASTRUNETBIA 2015-71942-REDT. One of the authors, Xidong Wang, wishes to thank China Scholarship Council (CSC) for financial assistance of a 4-year study at Universidad Politécnica de Madrid.

\section{ORCID}

Emiliano Pereira (iD) https://orcid.org/0000-0002-9029-1352

\section{REFERENCES}

1. Lu X, Ding K, Shi W, Weng D. Tuned mass dampers for human-induced vibration control of the Expo Culture Centre at the World Expo 2010 in Shanghai, China. Struct Eng Mech. 2012;43(5):607.

2. Basu B, Bursi OS, Casciati F, et al. An EACS joint perspective. Recent studies in civil structural control across Europe. Struct Control Health Monit. 2014;21(12):1414.

3. Lu Z, Chen X, Li X, Li P. Optimization and application of multiple tuned mass dampers in the vibration control of pedestrian bridges. Struct Eng Mech. 2017;62(1):55.

4. Casado CM, Díaz IM, de Sebastián J, Poncela AV, Lorenzana A. Implementation of passive and active vibration control on an in-service footbridge. Struct Control Health Monit. 2013;20(1):70.

5. Casciati F, Casciati S, Faravelli L. A contribution to the modelling of human induced excitation on pedestrian bridges. Struct Saf. 2017;66:51.

6. Díaz IM, Reynolds P. Robust saturated control of human-induced floor vibrations via a proof-mass actuator. Smart Mater Struct. 2009;18(12):125024.

7. Díaz IM, Reynolds P. Acceleration feedback control of human-induced floor vibrations. Eng Struct. 2010a;32(1):163.

8. Díaz IM, Pereira E, Reynolds P. Integral resonant control scheme for cancelling human-induced vibrations in light-weight pedestrian structures. Struct Control Health Monit. 2012;19(1):55.

9. Hanagan LM, Kulasekere EC, Walgama KS, Premaratne K. Optimal placement of actuators and sensors for floor vibration control. J Struc Eng. 2000;126(12):1380.

10. Pereira E, Díaz IM, Hudson EJ, Reynolds P. Optimal control-based methodology for active vibration control of pedestrian structures. Eng Struct. 2014;80:153.

11. Asami T, Nishihara $\mathrm{O}$, Baz A. Analytical solutions to $\mathrm{H}[\mathrm{sub} \infty]$ and $\mathrm{H}[\mathrm{sub} 2]$ optimization of dynamic vibration absorbers attached to damped linear systems. J Vib Acoust. 2002;124(2):284.

12. Warnitchai P, Hoang N. Optimal placement and tuning of multiple tuned mass dampers for suppressing multi-mode structural response. Smart Struct Syst. 2006;2(1):1.

13. Li Q, Fan J, Nie J, Li Q, Chen Y. Crowd-induced random vibration of footbridge and vibration control using multiple tuned mass dampers. J Sound Vib. 2010;329(19):4068.

14. Zuo L, Nayfeh SA. Minimax optimization of multi-degree-of-freedom tuned-mass dampers. J Sound Vib. 2004;272:893.

15. Mohtat A, Dehghan-Niri E. Generalized framework for robust design of tuned mass damper systems. J Sound Vib. 2011;330(5):902.

16. Salcedo-Sanz S, Camacho-Gómez C, Magdaleno A, Pereira E, Lorenzana A. Structures vibration control via Tuned Mass Dampers using a co-evolution Coral Reefs Optimization algorithm. J Sound Vib. 2017;393:62.

17. Chang CM, Shia S, Lai YA. Seismic design of passive tuned mass damper parameters using active control algorithm. $J$ Sound Vib. 2018;426:150.

18. Poncela A, Casado C, Baeyens E, Perán JR. Design of devices for protecting civil structures using fixed-order $\mathcal{H}$ control. Struct Control Health Monit. 2007;14(2):239.

19. Wang X, Díaz IM, Pereira E, Xu Q. Optimal TMD-based control for human-induced vibration. In: Inproceedings of the 3rd International Conference on Mechanical Models in Structural Engineering; 2015; Seville Spain.

20. Wang X, Díaz IM, Pereira E. MIMO control design including input-output frequency weighting for human-induced vibrations. In: Proceedings of the 6th European Conference on Structural Control. Sheffield; 2016; UK.

21. Gawronski WK. Advanced Structural Dynamics and Active Control of Structures. New York: Springer; 2004.

22. Wang X, Pereira E, Díaz IM, García-Palacios JH. Velocity feedback for controlling vertical vibrations of pedestrian-bridge crossing. Practical Guidelines. Smart Struct Syst. 2018;22(1):95.

23. Griggs WM, Anderson BD, Lanzon A. A "mixed" small gain and passivity theorem in the frequency domain. Syst Control Lett. 2007;56(9-10):596.

24. Díaz IM, Reynolds P. On-off nonlinear active control of floor vibrations. Mechanical Syst and Signal Processing. 2010b;24(6):1711-1726. 
25. Preumont A. Vibration Control of Active Structures. Netherlands: Springer; 2011.

26. http://apsdynamics.com/en/products/vibration-exciter/ball-bearing-exciter.html; 2018.

27. ISO 2631-1. Mechanical Vibration and shock- Evaluation of human exposure to whole-body vibration- Part 1- General Requirements; 1997.

28. ANSYS Multiphysics. Release 10.0, Help System, Ansys Elements Reference, ANSYS Inc; 2005.

29. Smith AL, Hicks SJ, Devine PJ. The Steel Construction Institute. Design of floors for vibration: A new approach (P354); 2007.

30. BS6841. Guide to measurement and evaluation of human exposure to wholebody mechanical vibration and repeated shock. British Standards Institute; 1997.

31. Lenzen KH. Vibration of steel joist-concrete slab floors. A.I.S.C Eng J. 1966;3(3):133.

32. Racić V, Pavić A, Brownjohn JMW. J Sound Vib. 2009;326(1-2):1.

33. Brownjohn JMW, Pavić A, Omenzetter P. A spectral density approach for modelling continuous vertical forces on pedestrian structures due to walking. Can J Civil Eng. 2004;31(1):65.

34. Murray TM, Allen DE. Ungar Design Guide 11, E. E. Vibrations of Steel-Framed Structural Systems Due to Human Activity. 2nd ed. USA: AISC; 2016.

35. Lagarias JC, Reeds JA, Wright MH, Wright PE, SIAM J. Convergence Properties of the Nelder-Mead Simplex Method in Low Dimensions. OPTIM. 1998;9(1):112.

36. ISO10137. Bases for design of structures-Serviceability of buildings and walkways against vibrations. New York: International Organization for Standardization; 2007.

How to cite this article: Wang X, Pereira E, García-Palacios JH, Diaz IM. A general vibration control methodology for human-induced vibrations. Struct Control Health Monit. 2019;e2406. https://doi.org/10.1002/stc.2406 